\title{
Constructivismo e intervención pedagógica: a propósito de quién construye
}

\author{
ANGEL GARCÍA DEL DUJO \\ Universidad de Salamanca
}

\begin{abstract}
SUMMARY.-Among the multiple questions that arise from the practical application, both from the professional and research point of view, of the constructivist principles inspiring educational reform, there is one whose answer makes a difference in the orientation of both the theoretical analysis and practical functioning of the educational process. The question we are referring to is who is doing the constructing? As opposed to the stances which would have the entire constructive activity fall on the internal control of the subject, the author defends the idea that there are different moments and spaces where constructive processes take place and there is always more than one constructor, which leads to the need for revising the concept of scaffolding.
\end{abstract}

\section{EL PROBLEMA Y SUS REPERCUSIONES}

Nos encontramos en uno de los momentos más álgidos del proceso de reforma educativa que se está intentando implantar en España. Ya en otras fases del proceso se escucharon opiniones, preguntas y reservas por parte de los profesores, profesionales, agentes sociales, instituciones, etc.; nos estamos refiriendo concretamente a las manifestaciones recogidas en el Informe-Síntesis ${ }^{1}$. Pero es ahora, cuando hay que implementar los primeros cursos de la reforma diseñada, cuando se avivan los ánimos y se agudiza la desazón, al menos la intelectual ${ }^{2}$. Las preguntas en este momento son muchas, muy diversas y vienen formuladas desde múltiples frentes.

Desde el colectivo de profesores que tienen que llevar a la práctica los principios constructivistas que inspiran la reforma los interrogantes son del siguiente tenor: si el aprendizaje consiste en un proceso de construcción del conocimiento y, lo que resulta más llamativo, el agente principal de esta operación es el alumno y cada alumno, ¿esta afirmación es válida para todo tipo, nivel y dominio de conocimiento? ¿Para el aprendi-

1. Ministerio de Educación y Ciencia. Debate del Diseño Curricular Base. Informe-Síntesis. Madrid, 1991.

2. Rojero, F. y Martín, A. «El conocimiento deseable», Signos. Teoría y práctica de la educación, 5-6 (1992) 5-15. Los autores se sorprenden de la facilidad con la que todo el mundo se define a sí mismo acríticamente como constructivista. 
zaje de las matemáticas y para el de las ciencias sociales?. ¿Qué tipo y nivel de construcción realiza el alumno en el aprendizaje de la división?. ¿Y en la adquisición del concepto de democracia?. ¿Qué es lo que construye el alumno: un (su) conocimiento o la manera de incorporar el conocimiento?. ¿Qué he de enseñarle: X o la forma de construir un (su) X? ¿Dónde tengo que poner el énfasis como profesor?, ¿Qué está demandando la sociedad: que el individuo resuelva los problemas, las situaciones o que sepa cómo abordarlos aunque los ejecute de una manera no correcta?. Y, sobre todo, ¿qué tengo que evaluar y cómo hacerlo?. Y ¿cuál es el papel del profesor?. ¿De guía?. ¿De ayuda?. ¿Maestro o peón de obra?. ¿Diseñador de estrategias que apoyen y fomenten la actividad constructiva del alumno, ese principio básico de la nueva Biblia de la educación?.

No hace mucho tiempo un profesor de la denominada ahora educación primaria nos hacía la siguiente reflexión: primero fue la enseñanza, luego el aprendizaje -con todas las variantes, combinaciones y redundancias que se quieran introducir; posteriormente el énfasis se traslada a la expresión «adquisición de conocimiento» y ahora se habla de construcción de conocimiento. Y añadía: esto del constructivismo ¿es una teoría o un método?. En el segundo caso ¿es un método con validez universal que no conoce límites por parte de la situación, ni de los objetivos pretendidos,....; y en el primero, es una teoría ¿de qué?. ¿De lo que se aprende, de cómo se aprende, de cómo hay que enseñar o es simplemente una teoría general del desarrollo humano con implicaciones obviamente a tener en cuenta en el proceso educativo de una persona?. Ante nuestra insistencia para una mayor concreción de esta última pregunta, agregó: entiendo que el constructivismo puede ser un marco adecuado para describir, explicar y prescribir el componente más netamente educativo del proceso de formación de una persona, pero no para aquella otra dimensión más orientada a la instrucción, al menos no para todo tipo de instrucción. Inmediatamente nos vinieron a la cabeza algunas controversias mantenidas con otros compañeros, incluso en el momento de escribir estas páginas; poco tiempo después volvíamos a releer el texto siguiente:

«If instructional designers design instruction, then constructivists are constructing something else. This «something else» may be a desirable educational intervention, but it does not appear to be instruction» ${ }^{3}$.

Hemos querido respetar el lenguaje de los profesionales, convencidos de su correspondencia-traducción al lenguaje de la investigación; en este sentido no cabe duda de que el cúmulo de interrogántes reseñados presentan suficiente calado como para conformar todo un programa de investigación interdisciplinar, programa que recoge cuestiones muy diversas aunque estrechamente interrelacionadas. Por la misma razón resulta impensable que en el marco de este artículo pretendamos responder a todas y cada una de las preguntas formuladas; pero antes de determinar cuál sea -y por qué- el objeto de nuestra reflexión queremos hacer un par de consideraciones generales. Primero, recordar una idea cuyas consecuencias pasan desapercibidas, a pesar de la frecuencia con la que se utiliza; nos referimos a la afirmación tantas veces reiterada de que en educación las cosas se presentan engañosamente simples, cuando en la realidad no lo son. Creemos que puede aplicarse al caso que nos ocupa; no puede interpretarse de otra manera la ex-

3. Dick, W. «An instructional designer's view of constructivism», en Educational Technology, 5 (1991), 41-44. 
cesiva alegría y premura con que se asignan funciones a los distintos elementos que intervienen en el proceso educativo dentro del marco teórico de referencia. Más aún, podría decirse que, siendo coherentes con dicho marco, una ubicación rígida de los elementos y una distribución simplista de funciones resultan incompatibles con un planteamiento constructivista. Las metáforas conllevan riesgos de deformación de visión también en educación y algunas de las preguntas formuladas aquí acusan esta deformación; otras, en cambio, denuncian la situación. En segundo lugar, aunque no referenciado por orden de importancia, la unidad de análisis en educación nunca puede ser un único elemento sino un sistema; solamente a posteriori, y una vez analizado en cuanto tal sistema, puede determinarse la preponderancia de uno u otro elemento o su mayor o menor sensibilidad a la hora de intervenir en el proceso. Lo contrario repugna a las tesis constructivistas, aunque no en apariencia, además de resituar al resto de elementos en lugares y con funciones cuando menos llamativas.

Aquí es precisamente donde pretendemos centrar nuestro análisis. Hay una pregunta no formulada en la relación anterior y que, sin embargo, está condicionando nuestro modo de ver las restantes cuestiones suscitadas: es la relativa a quién construye. Mejor dicho, aunque no está formulada directamente, sí que está respondida, al partir del supuesto de que el -único- agente responsable de la actividad constructiva del conocimiento es el alumno y, por ende, cada alumno. Precisamente este último añadido es el que motiva muchas de las dudas posteriores y otras que perfectamente podrían agregarse. Podría contestarse que en ningún momento se está pensando en prescindir de la presencia del profesor, pero esto no resuelve el problema ni llega al fondo de la cuestión. Depende de la función que se le asigne y mucho nos tememos que las funciones que normalmente se recogen en la literatura psicológica y pedagógica actual -de guía, de ayuda, de ajuste permanente de la misma, diseñador de estrategias, etc.- no van más allá de la periferia del proceso. Otra vez más la metáfora -el concepto de andamiaje- estaría cobrando su tributo. Estaríamos desembocando en planteamientos ya conocidos, si bien ahora enfocados al revés. Durante mucho tiempo fue el alumno el sujeto pasivo del proceso, aunque nunca se le negó un cierto nivel de actividad externa; la otra, la interna, se daba por desconocida o se negaba. Ahora es el profesor el que mantiene su actividad en el contorno del proceso, ayudando, ajustando, apoyando, fomentando... la actividad constructiva del alumno, para lo que diseña tareas, procedimientos, estrategias; se vuelve a pasar por alto su participación en el hecho mismo de construcción de conocimiento, o se reduce a actividades periféricas, acaso también por desconocimiento. Planteado en términos más teórico-pedagógicos, estamos en ambos casos ante ópticas unidireccionales y, por tanto, parciales de la naturaleza del proceso educativo, que no puede ser otra que la relación y acción mutua que se establece en el binomio medio-sujeto. El término «interacción» $-\mathrm{y}$ su espacio o dominio- entre el sujeto y lo otro (v. gr. el profesor) es uno de los terrenos que en educación necesitan mayor desarrollo e investigación, puesto que de su conocimiento están dependiendo muchos y fuertes problemas no resueltos tanto en educación formal como no formal ${ }^{4}$.

4. Puede verse a este respecto la obra de Newman, D.; Griffin, P. y Cole, M. La zona de construcción del conocimiento: trabajando por un cambio cognitivo en educación, Madrid, MEC. Ed. Morata, 1991. Toda su aportación se asienta en el concepto de interacción educativa, cuyo espacio identifica con la zona donde tiene lugar la actividad constructiva que provoca el cambio cognitivo. 
Con la intención de aclarar y concretar más nuestra propuesta, insertamos un texto suficientemente representativo de esa otra posición que estamos intentando matizar.

«Si se acepta que el aprendizaje escolar consiste, como postula el constructivismo, en un proceso de construcción de significados y atribución de sentidos cuya responsabilidad última corresponde al alumno y si se acepta, como se hace habitualmente desde esta perspectiva teórica, que en último término nadie puede sustituir al alumno en dicha empresa, entonces ¿cómo cabe entender la influencia educativa que trata de ejercer el profesor cuando enseña a sus alumnos? ¿cómo consigue el profesor enseñar lo que en definitiva el alumno debe construir por sí mismo?. Una posible respuesta a estas preguntas consiste en entender la influencia educativa en términos de ayuda prestada a la actividad constructiva del alumno; y la influencia educativa eficaz en términos de un ajuste constante y sostenido de esta ayuda a las vicisitudes del proceso de construcción que lleva a cabo el alumno» (La negrita es nuestra; la cursiva no) ${ }^{5}$.

No tratamos de suplantar al alumno en esa empresa, por supuesto, sino de advertir que no es exclusivamente suya en su totalidad. Antes -0 por debajo- de interrogarnos cómo entender y materializar la influencia educativa, hay una pregunta previa -o más profunda-: ¿en qué consiste y hasta dónde llega la acción de la influencia en educación?. Luego, las preguntas se multiplican en torno a la manera de canalizar dicha influencia.

Pues bien, en educación tal acción siempre es, al menos, bidireccional, recurrentemente progresiva y traspasa los límites de la mera disposición externa del conjunto de elementos que configuran una situación educativa. Ejemplificando el planteamiento en el contexto escolar, la ayuda pedagógica del profesor y el proceso de construcción del conocimiento del alumno no deben interpretarse como dos instancias distintas -de naturaleza- y distantes - una externa y otra interna- que tienen que encontrarse y adecuarse, sino que en realidad hay un solo fenómeno -la construcción de conocimiento- que se ejecuta mediante un proceso compartido y cooperativo en el que intervienen múltiples materiales e instrumentos, cuyo alto grado de ductilidad permite sucesivos tratamientos y transformaciones, y en ocasiones rupturas y/o desviaciones -positivas o negativas- de los objetivos así como de las vías contempladas en un primer y previsible diseño.

La recurrencia a símiles y metáforas traídas de otros ámbitos resulta inevitable en el estado en que se encuentra la investigación actual; no obstante entendemos que pueden apreciarse con claridad las repercusiones del enfoque que estamos formulando para el tratamiento de los múltiples problemas -y no sólo los de tipo educativo- que subyacen a los interrogantes con los que iniciábamos este trabajo. En este sentido nos inclinamos a pensar que el planteamiento propuesto termina conduciéndonos a un análisis diferente del fenómeno y proceso educativo. A continuación reseñamos algunas de estas consecuencias, sin ánimo de exhaustividad ni de desarrollar ampliamente cada punto, tarea en la que nos encontramos trabajando.

1. Si en verdad las inquietudes se despiertan, como parece desprenderse de las opiniones de los profesionales, al interpretar que quien construye es únicamente el alumno

5. Coll, C. «Constructivismo e intervención educativa: ¿cómo enseñar lo que se ha de construir?» en I Congreso Internacional de Psicología y Educación. Intervención psicoeducativa, Madrid, 1991. 
y que el proceso constructivo reside en la actividad interna que desarrolla el sujeto en solitario; si el origen de las numerosas dudas que se suscitan en torno a qué sea lo que se construye, su validez, la forma de comprobarlo, la manera de entender la acción educativa, etc., radica en la existencia y diversidad de las múltiples interpretaciones que hacen los distintos sujetos ante una misma situación, entonces hemos de concluir que todas esas preguntas se sitúan en un marco diferente desde el momento en que se acepta que el proceso constructivo es un proceso compartido, cooperativo $\mathrm{y}$, en consecuencia, negociado $^{6}$. Fijémonos solamente en dos cuestiones estrechamente conexionadas aunque bien diferentes a primera vista; una de orden filosófico y pedagógica la otra. La temida acusación de relativismo que se ha lanzado permanentemente contra un constructivismo de tipo solipsista se desvanece al ser corregido éste en la línea de que tanto la construcción de significados, la atribución de sentidos y usos como los procedimientos utilizados en su elaboración tienen un carácter público y compartido, inevitablemente social y cultural $^{7}$. Por otra parte, la acción educativa no puede reducirse ni verse solamente como un conjunto de dispositivos ofertados a la actividad constructiva, siendo aquélla externa y de dominio del agente educativo y ésta interna y propia del educando. La acción educativa no se limita ni comienza ni termina construyendo artefactos ${ }^{8}$ sino interpretando las primeras -o en su ausencia las hipotéticas- acciones y reacciones del sujeto, que no son otra cosa que manifestaciones de las interpretaciones que a su vez está llevando a cabo así como de la forma en que las realiza. Y en este juego de interpretaciones -actividad de ambos participantes- y de acciones -también de ambos-consiste el proceso de construcción de conocimiento, siendo ambas momentos parciales de un sólo fenómeno total $^{9}$. Hablamos de construcción de conocimiento, que no reproducción ni mera trans-

6. J. Bruner advertía hace unos años de los riesgos que se corren en educación al afrontar el estudio del niño como educando autónomo, «un agente solitario que conquista el mundo por su cuenta». Y añadía: «Lo que todavía nos falta es una teoría razonada de cómo debe interpretarse la negociación del significado lograda socialmente en cuanto axioma pedagógico, si bien ha comenzado a perfilarse en la obra de Vygotsky y en algunas teorías contemporáneas como las de M. Cole y H. Mehan». Bruner, J., Realidad mental y mundos posibles, Barcelona, Ed. Gedisa, 1988, p. 130.

7. Bruner, J. Actos de significado. Más allá de la revolución cognitiva, Madrid, Alianza Editorial, 1991 , pp. 38 y ss.

8. Llama la atención en este punto el concepto de «tarea» que manejan algunos autores, lo que supone una llamada de atención, a nuestro afán por diseñar paquetes de estrategias y procedimientos de antemano. «Nos equivocaremos si tratamos de especificar la tarea 0 , incluso, el conjunto de tareas en una determinada situación de aprendizaje y desarrollo. Lo que podemos tratar de especificar son los conjuntos de comprensiones que se negocian en cuanto objetivo de la tarea específica y el cambio de las limitaciones con el fin de acomodar el crecimiento cognitivo». Newman, D.; Griffin, P. y Cole, M., La zona de construcción del conocimiento, Madrid, MEC. Ed. Morata, 1991, p. 143. Para estos autores las tareas son ficciones estratégicas utilizadas por las personas como forma de negociar la interpretación de una situación y, por lo tanto, sometidas a variación en función del curso de la negociación, que afecta incluso a la definición de las tareas mismas, aunque a nosotros nos parezca que es misión propia y exclusiva del agente educativo.

9. La multiplicidad y diversidad de formas que pueden adoptar esas interacciones en sujetos diferentes ante un proceso educativo determinado permiten explicar la variabilidad de procedimientos y resultados, que ni deben ser considerados como puras y espontáneas invenciones de unos ni como efectos de diseños a priori de otros; estamos en presencia de un sistema y proceso abierto, en el que el juego de interacciones no se presenta ni cerrada ni ordenadamente. 
misión de conocimientos, aunque no cabe duda de que existe trasmisión de información y suele terminar habiendo una cierta correspondencia entre conocimientos ${ }^{10}$.

2. Adoptando ahora un punto de vista más general, puede encontrarse en el epígrafe anterior la alternativa a una situación muy habitual en educación que mantiene atenazados tanto al análisis teórico como la práctica educativa; nos referimos al hecho de considerar como instancias separadas lo uno y lo otro, el sujeto y el medio, ya se presente éste último directamente o a través de sus intermediarios. Esta es la situación que reflejan también las preguntas iniciales y en absoluto debe extrañar dentro de esa perspectiva el debate surgido en torno al reparto de papeles. Ambas instancias, a pesar de sus influencias mutuas aludidas por bonitos rótulos, aunque no concretadas en el funcionamiento práctico, presentan en la realidad un comportamiento relativamente independiente y autónomo, siendo susceptibles de diferentes tratamientos y modelos de análisis. Quizás sea ésta la fase en la que se encuentra la pedagogía del momento; el constructivismo para explicar el aprendizaje, algo que ocurre en el interior del sujeto, mientras la instrucción, esa envoltura que lo facilita, sigue aferrada a esquemas más propios de una física antigua $^{11}$. De nuevo estamos cayendo en separatismos y reduccionismos muy al uso en educación y, desde luego, no deja de ser otra forma más de expulsar a la educación y de tergiversar la naturaleza del proceso educativo. Newman, Griffin y Cole califican esta situación de falsa dicotomía y la hacen responsable de llevar a la teoría y a la práctica educativa a un callejón sin salida, al presentar lo externo, el medio como «fuerza no analizada que influye sobre el organismo individual» mientras todo el peso de la teoría constructivista se hace recaer sobre el dominio interno del sujeto ${ }^{12}$. Además encuentran muy extendida esta posición en los trabajos de investigación de ciencia cognitiva, a pe-

10. Aunque el planteamiento en sí no exige la uniformidad de las interpretaciones, sino más bien al contrario, sobre todo al principio de la interacción, en el decurso de la misma pueden presentarse y de hecho se producen aproximaciones sucesivas en virtud de la fuerza de uno de los elementos intervinientes, los factores sociales y culturales. «La asimetría de las relaciones sociales existentes entre un adulto experto y un niño novato proporciona la direccionalidad, no relativista del cambio en y desde los intercambios en la ZDP». Newman, D.; Griffin, P. y Cole, M., op. cit., p. 89. He aquí el difícil equilibrio y el riesgo de la acción educativa, hasta el punto de que una preponderancia absoluta de ese componente puede terminar arruinando la perspectiva constructivista y el propio concepto de educación. Pero donde está el riesgo, allí se encuentra la virtualidad; la mayor o menor ruptura o inclinación de ese difícil equilibrio por o hacia una u otra parte explicaría tanto las situaciones que conocemos como creativas como las de adoctrinamiento y, en definitiva, lo que podríamos llamar el mayor o menor grado de libertad y autonomía educativa.

11. Cfr. Aznar Minguet, P. «Paradigma tecnológico versus paradigma positivista: hacia una teoría constructivista del conocimiento pedagógico como fundamento de una teoría constructivista de la educación». Comunicación presentada al X Seminario Interuniversitario de Teoría de la Educación. Oviedo, 1991. La autora advierte que el marco teórico-metodológico propuesto «se aparta considerablemente de los planteamientos positivistas, hasta el punto de poder afirmar que cualquier reminiscencia positivista en el paradigma constructivista-tecnológico es más aparente que real».

12. Siempre nos ha llamado la atención a este respecto el contraste que se establece entre los resultados materializados en un examen escrito y aquéllos otros que se producen cuando se instrumenta un encuentro entre profesor y alumno mediante una entrevista de comentario y/o revisión de dicho examen. Con frecuencia encontramos en este segundo momento, si los factores emocionales no bloquean la situación, que aparecen notables diferencias entre unos y otros, cuando se solicita del alumno una ampliación o matización de un concepto, procedimiento, etc., previa interpretación por parte del profesor de lo que cree que aquél quiso decir en el primer momento. 
sar de los esfuerzos de Vygotsky por interpretar ambos polos como partes integrantes de un mismo proceso.

\section{SEGUNDA VISIÓN DEL MISMO PROBLEMA}

Los números 5 y 9 de la revista Educational Technology, correspondientes a los meses de mayo y septiembre de 1991, recogen una amplia reflexión de diversos autores que se consideran a sí mismo constructivistas sobre las repercusiones de este marco para la teoría y la práctica de la instrucción así como un interesante debate con otros autores más próximos a la tradición objetivista en educación, junto con posturas que asumen postulados intermedios. Nada mejor que una lectura pausada de las aportaciones que hacen los distintos autores para advertir no sólo ya las múltiples interpretaciones que se cobijan bajo el manto constructivista -lo contrario sería incompatible con la propia idea de constructivismo como señalan algunos-, ni siquiera la diversidad de prácticas educativas que circundan dicha idea, como indican otros, sino incluso la posibilidad de que estemos situándonos ante realidades educativas diferentes en función del lugar -los elementos y la forma de tratarlos- donde coloca el énfasis cada autor, de manera que terminemos realizando un análisis del proceso educativo distante de las exigencias que marca la propia propuesta teórica, v. gr., aún admitiendo todos la importancia del factor «contexto», no es lo mismo abordar su tratamiento e implementación como una parte y parcela de la dinámica del proceso educativo que hacerlo como un artefacto empaquetado previamente. $\mathrm{O}$, aún compartiendo todos que «the role of education in a constructivist view is to show students how to construct knowledge» (Cunningham, mayo 1991), no es lo mismo, en cuanto a la función que desempeña el alumno y el profesor, subrayar a continuación que «the constructivist would proceed by selecting tasks that are relevant to the child's lived experience... the teacher then provides access to tools that can be used to better understand or construct solutions to the problem», que entender que «the plans -the principles, rules, procedures we teach- are part of the subject matter of purposeful action, not something to be unproved upon or transformed into axiomatic theories of action» (Duffy and Jonassen, mayo 1991); la segunda interpretación no sólo afecta a lo que se construye, a la vez que destaca los distintos momentos en que tiene lugar un proceso constructivo, sino también, y sobre todo, al papel que han de jugar los agentes intervinientes en el proceso. Pero antes de centrarnos en este punto, que viene siendo el objeto de nuestro estudio en la intención más amplia de enfocar adecuadamente el análisis del proceso educativo en esta perspectiva, queremos hacer también ahora algunas consideraciones.

Primeramente, resaltar la coincidencia de los numerosos interrogantes que aparecen diseminados a lo largo de los artículos respecto de aquéllos otros que venían formulados por parte de los profesionales; también aquí surgen dudas en torno a si todo conocimiento puede ser construido por los alumnos (Winn, septiembre 1991), si el constructivismo es igualmente útil en todas las situaciones o si, siendo apropiado para algunos tipos de aprendizaje en un entorno educativo general, resulta perjudicial para solucionar nuestros problemas educativos más urgentes (Merrill, mayo 1991), si es igualmente aplicable en todas las áreas y para todos los tópicos (Molenda, septiembre 1991), si en realidad estamos refiriéndonos a habilidades de orden superior o este enfoque es válido para todo ti- 
po y dominio de aprendizaje (Dick, mayo 1991), si es posible construir habilidades y estrategias independientemente de los problemas a que son aplicables (Cunnigham, mayo 1991); dudas en torno a si el grado de autonomía del alumno en la construcción del conocimiento, incluido el relativo al desarrollo de estrategias y la determinación de objetivos, no dificulta, si no imposibilita, la planificación de actividades de instrucción (Winn, septiembre 1991); dudas en torno a la posibilidad y a los criterios para desarrollar la evoluación y la necesidad de reconceptualizar socialmente los objetivos de la educación para evitar disonancias entre las expectativas sociales, más afines a un planteamiento objetivista, y los objetivos manifestados y perseguidos por las instancias educativas (Jonassen, septiembre 1991).

En segundo lugar, queremos llamar la atención sobre la existencia de un ulterior nivel de interrogantes que se apoya en, a la vez que trasciende, las dudas manifestadas más arriba y que, a nuestro entender y para nuestro objetivo, permite situar el problema en sus términos precisos. A fuerza de dar vueltas a las distintas posiciones que puede adoptar el constructivismo -más o menos radicales, un constructivismo moderado o extremo- y de contraponer sus puntos de vista respecto de aquéllos otros que se derivan de paradigmas más tradicionales, algunos autores terminan preguntando no ya sobre la utilidad y/o validez, sino algo más allá que afecta a la propia naturaleza de la propuesta, aunque aparentemente se presente como una cuestión de simple extensión o nivel de aplicabilidad: ies realmente una teoría o es una estrategia instruccional para un tipo particular de resultados de aprendizaje? (Dick, mayo 1991). Y aunque desde el constructivismo no se duda en presentarlo como una perspectiva pedagógica alternativa que puede ser aplicada para la mayor parte si no todos los objetivos de aprendizaje (Duffy and Jonassen, septiembre 1991), ello no significa que se haya agotado la serie de interrogantes: ¿en qué termina lo que en principio aparece como una teoría del aprendizaje?. ¿En mera teoría del curriculum, puesto que las decisiones conciernen más a «what to teach than how to teach it», sobre todo en el constructivismo extremo (Reigeluth, septiembre 1991) o en teoría de la instrucción que afecta fundamentalmente a la coreografía del proceso de enseñanza-aprendizaje (Perkins, mayo 1991). Hemos de reconocer el gran acierto de estas últimas preguntas, pues no son pocas las ocasiones ni los autores cuyas reflexiones terminan orientándose predominantemente en uno $u$ otro sentido, $y$ a veces con resultados difíciles de asumir dentro de las exigencias de la propia perspectiva; claro, que el debate aquí afecta a todos los elementos intervinientes y objetivos, contenidos, procedimientos, etc. terminan por entremezclarse y resituarse de una u otra manera en función de la perspectiva de cada autor, hasta el punto de que con frecuencia no resulta fácil el mutuo entendimiento -ya decíamos al principio que las cosas en educación se presentan engañosamente simples-. Pero esto no significa otra cosa distinta que admitir que, más allá de lo uno o de lo otro, lo primero que permite, proporciona y exige el planteamiento constructivista es un análisis global y diferente del fenómeno y proceso educativo -teoría de la educación ${ }^{13}$ con fuertes implicaciones para la práctica educativa, la instrucción

13. Estamos de acuerdo con la caracterización que Newman, D.; Griffin, P. y Cole, M. hacen de la investigación sobre la educación como ciencia de lo artificial, en el sentido de Herbert Simon (1979): «el estudio de cómo operan las interacciones educativas es inseparable de la tarea de rediseñarlas para que funcionen mejor». Newman, D.; Griffin, P. y Cole, M., op. cit., p. 154. Y esta forma de entender la educación y su conocimiento es consistente con el llamado enfoque tecnológico de la educación -referi- 
y hasta para el mismo concepto de educación. Lo contrario conlleva introducir serias distorsiones en el propio planteamiento, como trataremos de demostrar a continuación.

Uno de los elementos al que todo enfoque constructivista concede mayor relieve viene dado por el entorno o contexto donde ocurre el aprendizaje; en la intención de evitar el denominado conocimiento descontextualizado e inerte como contraposición a lo que constituye el objetivo básico del constructivismo definido en términos de comprensión y uso activo del conocimiento, todos los autores afrontan el análisis de esta variable mediante una amplia descripción de las características exigibles que suele terminar en la calificación de relevante, útil, significativo, real, auténtico, etc. Si el conocimiento en cada sujeto consiste en la atribución de sentido y uso de la información en función de sus propios esquemas interpretativos, parece lógico pensar que, cuanto más rico, útil... sea el contexto en el que se presenta la información, mayor posibilidad y facilidad tendrá el sujeto para poner en marcha las complejas operaciones que lleva consigo el término conocimiento en este enfoque.

A continuación los distintos autores introducen variantes en el principio que acabamos de exponer sucintamente, buscando siempre la forma más adecuada y rentable de diseñar y presentar el contexto al sujeto. Así, mientras unos defienden la necesidad de contar con múltiples y flexibles entornos de aprendizaje por entender que, en dominios complejos y mal estructurados y a nivel de conocimiento avanzado, donde resulta más apropiada la aplicación del constructivismo (Spiro, Feltovich, Jacobson y Coulson, mayo 1991; Jonassen, septiembre 1991), el significado de un concepto no se agota en una sola representación o esquema sino que debe ser interpretado desde diferentes perspectivas situacionales, obteniendo como resultado diferentes perspectivas conceptuales en función de la variedad de usos, otros apoyan la idea del aprendizaje generativo dentro de un macrocontexto (Cognition and Technology Group, mayo 1991) y otros, por fin enfatizan la propuesta de trabajo en grupo de manera que cada sujeto pueda comparar diversas interpretaciones en términos de su uso (Cunningham, mayo 1991).

Hasta aquí podríamos estar de acuerdo en que hay pocas cosas que objetar; tan sólo recordar que la preocupación por la descontextualización del aprendizaje no es exclusiva del constructivismo y que desde siempre se han utilizado ejemplos y contraejemplos como aproximación básica al aprendizaje de conceptos (Dick, septiembre 1991). El problema aparece cuando se asigna al profesor el papel de seleccionador, confeccionador y/o diseñador de contextos, como parece desprenderse de las posiciones que mantienen los autores, limitando así su función a la disposición y puesta en escena del conjunto de elementos que configuran el contexto de aprendizaje, incluidas las herramientas o estra-

do al conjunto del proceso pedagógico-, que a su vez resulta compatible con los paradigmas más recientes a los que se quiere someter el aprendizaje y la educación, v. gr. el constructivismo. Lo que no admite este marco teórico, como reiteradamente apuntamos en este trabajo, es una concepción tecnológica interpretada restrictivamente en el sentido meramente aplicativo de recetas de validez universal en esta o aquella acción didáctica. Sarramona, J., Tecnología educativa. Una valoración crítica. Barcelona, Ceac, 1990. Idem: «Concepció actual de la tecnología de l'educació», Crónica d'ensenvament, 22 (1990) 4-5. Colom Cañellas, A. J., «El saber de la Teoría de la Educación. Su ubicación conceptual», en este mismo número. De esta manera desaparece la contradicción señalada críticamente por Rojero, F. y Martín, A. «El conocimiento deseable», Signos. Teoría y práctica de la educación, 5-6 (1992) 5-15. 
tegias que pueden ser usadas para una mejor comprensión o construcción del problema (Cunningham, mayo 1991). Enfocar el análisis del proceso educativo con esta óptica y en estos términos es reducir el marco de la influencia de la acción educativa, abrir una brecha entre ésta y la actividad del sujeto interpretando que ambas se corresponden con sendas funciones distribuidas entre los protagonistas del proceso, cuando en realidad se desarrollan juntas en cada uno de ellos; es, en resumen, distinguir entre lo interno y lo externo y someterlos a tratamientos diferentes.

Estamos más de acuerdo a este respecto con las matizaciones introducidas por W. D. Winn (septiembre 1991). Consciente de las dificultades que encierra hablar de diseño instruccional y de prescripción de estrategias instruccionales dentro del constructivismo, advierte que, si «traditionally... both content and strategy are therefore imposed on the student from the outside», el constructivismo exige «that the selection of strategies, and even of content, is now shifted to the moment a student learns and is not decidet ahead of time by a designer». Para nosotros no solamente se trata de un mero cambio de sitio o de momento respecto de la toma de decisiones, sino que las implicaciones van más allá: se trata de reconocer la presencia y participación del agente educativo, v. gr. el profesor, en el propio proceso de construcción de conocimiento del alumno, hasta el punto de admitirle como co-constructor de dicho proceso y del consiguiente conocimiento. Su participación no queda en lo periférico -el diseño y/o la implementación realizada por otros de acciones educativas o de «shells» en expresión del autor- sino que penetra en la propia actividad del sujeto, a través de cuyas manifestaciones se apropia de la misma y la devuelve una vez reinterpretada. Parafraseando el autor, el efecto del cambio de sitio es reintegrar «lo externo» en la misma y única dinámica del proceso educativo y, por supuesto, «esto es consistente con las posiciones que defienden que el pensamiento no puede ser separado de la acción», referidas ambas dimensiones tanto al alumno como al profesor y al desenvolvimiento de ambos en el proceso. Por último, el resultado no sólo es «that instruction and performance are de-emphasized by constructuvists», para colocarlo «on learning» sino que lo que queda resaltado sobre manera es el componente educativo del proceso, haciendo presente y posible la reflexión del sujeto sobre el propio proceso, dándole sentido y uso.

\section{CONCLUSION AL MISMO NIVEL O LA NECESIDAD DE REVISAR EL CONCEPTO DE ANDAMIAJE}

Dice J. Bruner que «en ninguno de los escritos de Vygotsky aparece ninguna expresión concreta sobre lo que quiere decir con ese andamiaje» (Brunner, 1988). Lo cierto es que tampoco él logra, cuando intenta reconstruir las intenciones de éste, clarificar definitivamente «el significado de este intrigante concepto». Apoyándose en otras múltiples metáforas, pretende describir lo que ocurre en la zona de desarrollo próxima y lo presenta como la sustitución temporal de la conciencia del alumno por la del instructor, en la misma línea que Vygotsky había señalado para la adquisición del lenguaje. Observando la relación que se establece entre madre y niño a propósito de la adquisición del lenguaje, situación permanentemente analizada y recuperada por otros muchos autores. Brunner advierte que la madre plantea «pequeños formatos», «rituales», «secuencias», «regu- 
laridades», etc. y añade: «esta secuencia proporciona un andamiaje para la referencia de la enseñanza» ${ }^{14}$.

Poco a poco y en nuestros días, el concepto de andamiaje ha ido materializándose, concretándose -escorándose, diríamos mejor- en una línea derivada de la fuerza y del ámbito de la propia metáfora, hasta el punto de que el significado más generalizado de este término en la literatura actual se refiere al conjunto de elementos externos -montables y desmontables, pero consistentes- diseñados y utilizados por el profesor para ayudar, provocar, posibilitar, fomentar... la actividad, interna por supuesto, del alumno relativa a la construcción de parte o todo un edificio -conceptos, procedimientos, valores-. A este respecto nos resulta inevitable inservar la muy acertada visión de $\mathrm{S}$. H. White, aunque esté formulada en sentido negativo:

«La zona de desarrollo próximo es algo más que el apoyo social que algunos llaman hoy día andamiaje; no se trata de un conjunto de dispositivos utilizados por una persona para apoyar una actividad de alto nivel realizada por otra» ${ }^{15}$.

La concepción actual de andamiaje pone el acento en el diseño e implementación de una serie de recursos utilizados como prótesis mientras aparece y/o se consolida una estructura o función. Y por más que se pretenda su anclaje en el contenido y en el contexto, la metáfora no logra erradicar una cierta imagen de estatismo en el comportamiento de aquellos y hasta de mecanicismo en su transmisión; las estructuras y los procedimientos no son algo que está ahí para su utilización de inmediato. En el fondo se está olvidando que lo que se andamia no es la actividad interna de un alumno sino la interacción entre profesor y alumno, que las acciones de aquél tienen como referencia, además de la actividad de éste, su propio sistema de actividad al que se han sometido las acciones o manifestaciones del alumno. Y, en resumen, que hay múltiples «momentos» y «lugares» donde tienen lugar procesos constructivos y siempre más de un constructor. Si el andamiaje se corresponde con la interacción, la zona de desarrollo próximo aparece como el núcleo de la educación.

\section{BIBLIOGRAFIA}

AzNAR Minguel, P., «Paradigma tecnológico versus paradigma positivista: hacia una teoría constructivista del conocimiento pedagógico como fundamento de una teoría constructivista de la educación», en X Seminario Interuniversitario de Teoría de la Educación. Oviedo, 1991.

BRUNER, J., Acción, pensamiento y lenguaje. Madrid, Alianza Editorial, 1984.

IDEM., Realidad mental y mundos posibles. Barcelona, Ed. Gedisa, 1988.

14. Nos estamos refiriendo a la obra de Bruner, J., Realidad mental... op. cit., pp. 83 y ss. En otros muchos escritos del autor puede rastrearse el concepto de formato y de andamiaje; véase en este sentido «La intención en la estructura de la acción y de la interacción» y «Los formatos de la adquisición del lenguaje» recopilados por J. L. Linaza en Bruner, J., Acción, pensamiento y lenguaje. Madrid, Alianza Editorial, 1984. Bruner, J. y Haste, H., La elaboración de sentido. La construcción del mundo por el niño. Barcelona, Paidós, 1990.

15. White, S. H., «Prólogo» a la obra de Newman, D.; Griffin, P. y Cole, M., op. cit., p. 14. 
IDEM., Actos de significado. Más allá de la revolución cognitiva. Madrid, Alianza Editorial, 1991.

BRUNER, J. y HASTE, H., La elaboración de sentido. La construcción del mundo por el niño. Barcelona, Paidós, 1990.

Cognition AND Technology Group at VANDERBILt University, «Technology and the design of generative learning environments», Educational Technology, 5 (1991) 34-40.

Coll, C., «Constructivismo e intervención educativa: ¿cómo enseñar lo que se ha de construir?», en I Congreso Internacional de Psicología y Educación. Intervención psicoeducativa. Madrid, 1991.

CunNingham, D. J., «Assessing constructions and constructing assessments: a dialogue», Educational Technology 5 (1991) 13-17.

DICK, W., «An instructional designer's view of constructivism», Educational Technology 5 (1991) 41-44.

DuFFY, T. M. y Jonassen, D. H., «Constructivism: new implications for instructional technology?», Educational Technology 5 (1991) 7-11.

IDEM., «Continuing the dialogue: an introduction to this special issue», Educational Technology, 9 (1991) 9-11.

GIL, P. y SANCHEZ, N., «Concepciones sobre el desarrollo y el aprendizaje», Signos. Teoría y práctica de la educación, 5-6 (1992) 25-35.

JONASSEN, D. H., «Evaluating constructivists learning», Educational Technology, 9 (1991) 28-33.

Merrill, M. D., «Constructivism and instructional design», Educational Technology 5 (1991) 45-53.

Molenda, M., «A philosophical critique of the claims of constructivism», Educational Technology 9 (1991) 44-48.

Monereo, C., Aprender a aprender y a pensar en la escuela. Actas de las Primeras Jornadas de Estudio sobre Estrategias de Aprendizaje. Madrid, 1990.

Newman, D., GRIFFIN, P. y COLE, M., La zona de construcción del conocimiento: trabajando por un cambio cognitivo en educación. Madrid, MEC-Ed. Morata, 1991.

Novack, J. D., «Ayudar a los alumnos a aprender cómo aprender. La opinión de un profesor investigador», Enseñanza de las ciencias 3 (1991) 215-228.

Novak, J. D. y GowIn, D., Aprendiendo a aprender. Barcelona, Martínez Roca, 1988.

PERKINS, D. N., «Technology meets constructivism: do they make a marriage?», Educational Technology, 5 (1991) 18-23.

REIGELUTH, Ch. M., «Reflections on the implications of constructivism for educational technology», Educational Technology, 9 (1991) 34-37.

Rojero, F. y MARTIN, A., «El conocimiento deseable», Signos. Teoría y práctica de la educación, 5-6 (1992) 5-15.

SARRAMONA, J., Tecnología educativa. Una valoración crítica. Barcelona, CEAC, 1990.

IDEM., «Concepció actual de la tecnología de l'educació», Crónica d'ensenyament 22 (1990) 4-5.

Spiro, R. J., Feltovich, P. J., Jacobson, M. J. y Coulson, R. L., «Cognitive flexibility, constructivism and hypertext: random access instruction for advanced knowledge acquisition in ill-structured domains», Educational Technology, 5 (1991) 24-33.

WINN, W. S., «The assumptions of constructivism and instructional design», Educational Technology 9 (1991) 38-40. 